\title{
Dense dispersed shear bands in gradient-structured $\mathrm{Ni}$
}

\author{
Yanfei Wang ${ }^{\mathrm{a}, \mathrm{b}}$, Chongxiang Huang ${ }^{\mathrm{a}, *}$, Yusheng $\mathrm{Li}^{\mathrm{c}}$, Fengjiao Guo ${ }^{\mathrm{a}}$, Qiong $\mathrm{He}^{\mathrm{a}}$, \\ Mingsai Wang ${ }^{\mathrm{a}}$, Xiaolei Wu ${ }^{\mathrm{d}}$, Ronald O. Scattergood ${ }^{\mathrm{b}}$, Yuntian $\mathrm{Zhu}^{\mathrm{b}, \mathrm{c}, * *}$ \\ ${ }^{\text {a }}$ School of Aeronautics and Astronautics, Sichuan University, Chengdu, 610065, China \\ ${ }^{\mathrm{b}}$ Department of Materials Science and Engineering, North Carolina State University, Raleigh, NC, 27695, USA \\ ${ }^{\mathrm{c}}$ Nano and Heterogeneous Structural Materials Center, School of Materials Science and Engineering, Nanjing University of Science and Technology, \\ Nanjing, 210094, China \\ ${ }^{\mathrm{d}}$ State Key Laboratory of Nonlinear Mechanics, Institute of Mechanics, Chinese Academy of Sciences, Beijing, 100190, China
}

\section{A R T I C L E I N F O}

\section{Keywords:}

Gradient structure

Shear bands

Ductility

Synergistic constraint

Strength heterogeneity

Nanostructured metal

\begin{abstract}
A B S T R A C T
During tensile deformation, nanostructured (NS) metals often fail soon after yielding by forming a localized shear band. Here we report the observation of high density of shear bands that are homogeneously dispersed in the NS layer of a gradient Ni sample. These shear bands were nucleated at early elastic/plastic strain stage, reached number saturation at $\sim 3 \%$ strain, and remained arrested by the central coarse-grained (CG) matrix during the entire plastic deformation, resulting in a uniform tensile plasticity comparable to that of CG matrix. The formation of dispersed shear bands was promoted by the elastic/plastic interaction between NS surface layer and CG matrix, and affected by the surface roughness and the hardness variation in the NS surface layer. The width of shear bands remained constant, but the intensity of strain accumulation increased almost linearly with applied tensile strain, suggesting a stable shear banding process. Microstructure examination revealed that the strain in shear bands was accommodated by mechanically driven grain boundary migration and grain coarsening. These results clarify the fundamental questions: why/how does the NS layer supported by CG matrix achieve large uniform elongation? Moreover, the findings demonstrate the possibility of activating dispersed stable shear bands by synthesizing gradient architecture for optimized mechanical performances, i.e., a new strategy for evading the strength-ductility tradeoff in NS metals.
\end{abstract}

\section{Introduction}

Gradient-structured materials with increasing grain sizes from nanostructured (NS) surface layers to coarse-grained (CG) central layer have attracted intensive interests due to their superior combination of strength and ductility (Wu et al., 2014a, 2016; Cheng et al., 2018, 2014b; Wu and Zhu, 2017; Lu, 2014; Fang et al., 2011; Wei et al., 2014; Lin et al., 2018; Zhu et al., 2019; Wang et al., 2018a; Lu et al., 2019). They were found to have synergistic strengthening to produce yield strength higher than what is predicted by the rule of mixture (Cheng et al., 2018; Wang et al., 2018a; Wu et al., 2014b) and extra strain hardening (Li et al., 2017; Wu et al., 2014a) to retain good ductility.

The mechanical incompatibility between different layers is believed responsible for the observed mechanical behavior and superior properties (Wu et al., 2014a; Wu and Zhu, 2017). Specifically, during tensile testing the softer CG layer yields first to start

\footnotetext{
${ }^{*}$ Corresponding author.

${ }^{* *}$ Corresponding author. Department of Materials Science and Engineering, North Carolina State University, Raleigh, NC, 27695, USA.

E-mail addresses: chxhuang@scu.edu.cn (C. Huang), ytzhu@ncsu.edu (Y. Zhu).
} 
plastic deformation, creating plastic/elastic interfaces where geometrically necessary dislocations are piled-up to create back-stress strengthening and extra work-hardening (Cheng et al., 2018; Kassner et al., 2013; Ming et al., 2019; Wang et al., 2018b; Yang et al., 2016; Zhu and Wu, 2019). When the whole sample is plastically deforming, the NS surface layers start unstable necking first, causing larger lateral shrinking in the NS layers than in the CG layer. This converts the applied uniaxial strain into multiaxial strains, which further helps with activating multiple slip systems and promoting dislocation hardening (Asaro, 1983; Wu et al., 2014a). In addition, residual stress produced during the processing of the gradient structure was also found to enhance mechanical properties (Moon et al., 2019; Yang et al., 2019).

A question arises on how the NS layer deforms in a gradient-structured sample. This question is critical for understanding the deformation mechanism and mechanical behavior of gradient materials. When deformed alone, NS metals often quickly start strain localization after yielding without much uniform elongation due to their low stain hardening capability (Ovid'ko et al., 2018; Valiev et al., 2016; Zhu and Wu, 2018). In gradient materials produced by surface mechanical attrition treatment, the NS layers have been reported to sustain very high plastic strains without apparent failure (Wu et al., 2014a, 2016; Cheng et al., 2018; Lu, 2014; Fang et al., 2011; Wei et al., 2014; Lin et al., 2018; Zhu et al., 2019). For a long time it was believed that the NS layers were deformed uniformly due to the constraint and support by the CG central layer (Fang et al., 2011; Lu, 2014; Wei et al., 2014; Wu et al., 2014a). However, it was recently reported that large shear bands were formed in the NS layer of gradient IF steel, which was delocalized along the gauge length to develop into a large strain accumulation zone with increasing tensile strain (Yuan et al., 2019). The shear bands accommodated the majority of applied tensile strain and provided some strain hardening to help with improving the overall strain hardening and ductility. This finding is surprising, because in both homogeneous NS and work-hardened polycrystalline materials the formation of such macroscopic SBs generally indicates the development of catastrophic strain localization, which will induce early fracture (Cheng et al., 2005; Ovid'ko et al., 2018; Yang et al., 2010). But it is not clear whether such stable shear banding is a universal behavior of the NS layers in gradient materials.

Shear banding is shear strain localization in a narrow zone, which often runs across multiple grain boundaries and twin boundaries during plastic deformation of polycrystalline metals. It is caused by local strain instability and accompanied with dramatic local orientation and texture change (Hong et al., 2010; Jia et al., 2013, 2012). Strain hardening was believed necessary to prevent shear banding (Borg, 2007; Mahesh, 2006). Nanostructured materials are especially prone to shear banding due to their low strain hardening capability. In the gradient structured metals, shear bands may be initiated in the NS layers. However, it may be difficult for the shear bands to propagate through the thickness/cross-section of the sample because the central CG layer typically has much higher strain hardening capability, which may act to stabilize the shear bands (Yuan et al., 2019). This needs to be further studied.

In this study we systematically studied shear band formation in the NS layer of gradient structured Ni plate with a large thickness of $3.6 \mathrm{~mm}$ using in-situ digital image correlation (DIC) technique. Nanostructured layers and microstructure gradients were produced with different processing techniques and parameters to study the mechanism of shear band nucleation and growth. It is found that high density of uniformly dispersed shear bands was formed instead of individual catastrophic shear bands that are typically reported in conventional homogeneous polycrystalline samples. Shear banding appears to be a primary mechanism for the NS layers to accommodate large applied plastic strain instead of being a pre-failure phenomenon.

\section{Experimental procedures}

\subsection{Materials and processing}

Commercial-pure Ni (99.60 wt\%) plates with a dimension of $100 \mathrm{~mm} \times 90 \mathrm{~mm} \times 3.6 \mathrm{~mm}$ were used for this study. The Ni plates were firstly annealed in vacuum at $750{ }^{\circ} \mathrm{C}$ for $4 \mathrm{~h}$, forming a fully recrystallized CG structure. Gradient plates with different surface roughness were symmetrically processed by means of rotationally accelerated shot peening (RASP) and piezoelectric surface nanocrystallization (PSNC) on both sides of as-annealed plates. RASP is a new surface mechanical attrition technique, which can vary both the speed and diameter of impacting balls (Wang et al., 2017). PSNC has relatively low processing efficiency but can produce extremely smooth NS surfaces (Li et al., 2016). Table 1 lists the main processing parameters for three different types of gradient samples $\mathrm{Ni}_{\mathrm{RASP}-\phi 2}, \mathrm{Ni}_{\mathrm{RASP}-\phi 1}$ and $\mathrm{Ni}_{\mathrm{PSNC}}$. In the RASP process, the samples peened by big balls are further treated using small balls for a longer time in order to reduce surface roughness.

\section{Table 1}

The processing parameters and referential label of gradient samples, where $\phi$ is the ball diameter, $v$ is velocity, $t$ is the processing time in RASP, and $d$ is the penetration depth of the indenter in PSNC.

\begin{tabular}{|c|c|c|c|c|c|c|c|}
\hline \multirow[t]{2}{*}{ Sample } & \multicolumn{3}{|c|}{ RASP step I } & \multicolumn{3}{|c|}{ RASP step II } & \multirow{2}{*}{$\frac{\text { PSNC }}{d, \mathrm{~mm}}$} \\
\hline & $\phi, \mathrm{mm}$ & $v, \mathrm{~m} / \mathrm{s}$ & $t, \min$ & $\phi, \mathrm{mm}$ & $v, \mathrm{~m} / \mathrm{s}$ & $t, \min$ & \\
\hline $\mathrm{Ni}_{\text {RASP }-\phi 2}$ & 2 & 40 & 5 & 0.5 & 40 & 10 & - \\
\hline $\mathrm{Ni}_{\text {RASP }-\phi 1}$ & 1 & 40 & 5 & 0.5 & 40 & 10 & - \\
\hline $\mathrm{Ni}_{\text {PSNC }}$ & - & - & - & - & - & - & 0.3 \\
\hline
\end{tabular}



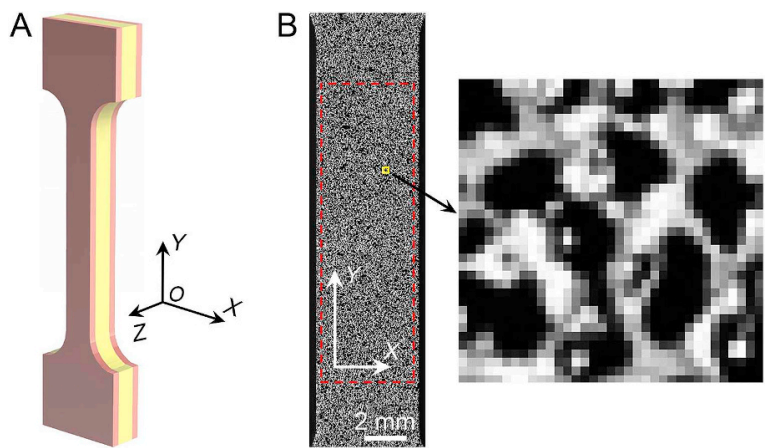

Fig. 1. (A) Illustration of the geometry of gradient tensile specimen. In the coordinate, $Y$ is the tensile loading direction, $X$ is the sample width direction, and $Z$ is the sample thickness direction with microstructure gradient. The $X O Y$ plane parallels to the NS surface. (B) Typical speckle pattern (the left subgraph) and detailed distribution of gray scale (the right subgraph) on the NS surface on gauge section. The red frame marks the $1452 \times 450$ pixel $^{2}$ effective calculation area.

\subsection{Microstructural characterization and mechanical tests}

The microstructures of gradient samples were characterized using scanning electron microscopy (SEM) and transmission electron microscopy (TEM). Focused ion beam was used to extract TEM foils precisely from selected positions. A Ni coating was deposited on the surface to protect the microstructure of topmost layer before ion beam cutting. TEM observation was performed in an FEI Tecnai G2 T20 microscope at $200 \mathrm{KV}$.

The variation of Vickers hardness along the depth was measured using a standard pyramid indenter at a load of $25 \mathrm{~g}$ for $15 \mathrm{~s}$. Tests for each sample were repeated at four independent locations. Indentations were arranged along a zigzag line, and the space between neighboring indentations was three times longer than the diagonal of impression. Dog-bone shaped tensile specimens with a gauge dimension of $18 \times 4.9 \times 3.6 \mathrm{~mm}^{3}$ were machined from as-processed gradient plates. Fig. $1 A$ illustrates the geometry of gradient tensile specimen. Tensile specimens with only the topmost $400-\mu \mathrm{m}$-thick layer or the central 2.0 -mm-thick core layer of $\mathrm{Ni}_{\mathrm{RASP}-\phi 1}$ material were prepared by polishing away the other layers of gradient tensile specimen. All tensile tests were performed at a strain rate of $5 \times 10^{-4} \mathrm{~s}^{-1}$.

\subsection{DIC strain characterization}

The strain distribution and evolution on the nanostructured surface and the lateral surface (the surface parallel to the $Y O Z$ plane) of gradient tensile specimens during tension were in-situly recorded by DIC, using a short-focus optical lens. A random speckle pattern was prepared by spraying black paints on white background before performing DIC imaging. Fig. 1B presents a typical speckle image taken from the nanostructured surface. As indicated by the dotted red frame, an effective area of $1452 \times 450$ pixel $^{2}$ with a resolution of $9.7 \mu \mathrm{m} /$ pixel was used for DIC calculation. The right subgraph shows the distribution of gray scale in a representative correlation window $\left(36 \times 36 \mathrm{pixel}^{2}\right)$. The mean intensity gradient of the gray profile of present speckle pattern is calculated as 48.6 , meaning a high correlation coefficient for strain calculation (Pan et al., 2010).

\section{Results}

\subsection{Surface roughness of the gradient samples}

Fig. 2 shows the linear distribution of the surface roughness of the gradient samples, which were measured using a white light interferometry. The height resolution is $\sim 20 \mathrm{~nm}$. The maximum height difference between the convex peak and concave valley of

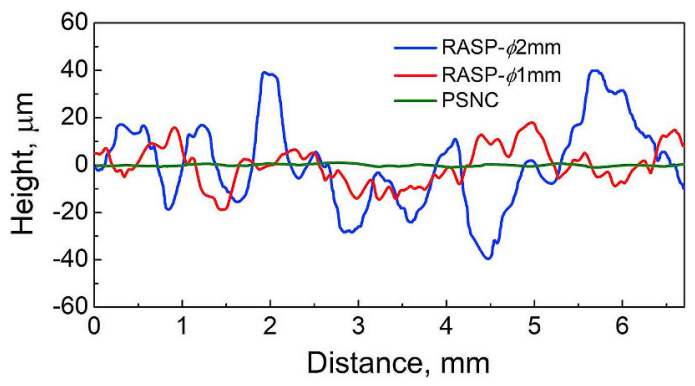

Fig. 2. The linear distribution of surface roughness measured in the three types of gradient samples. 

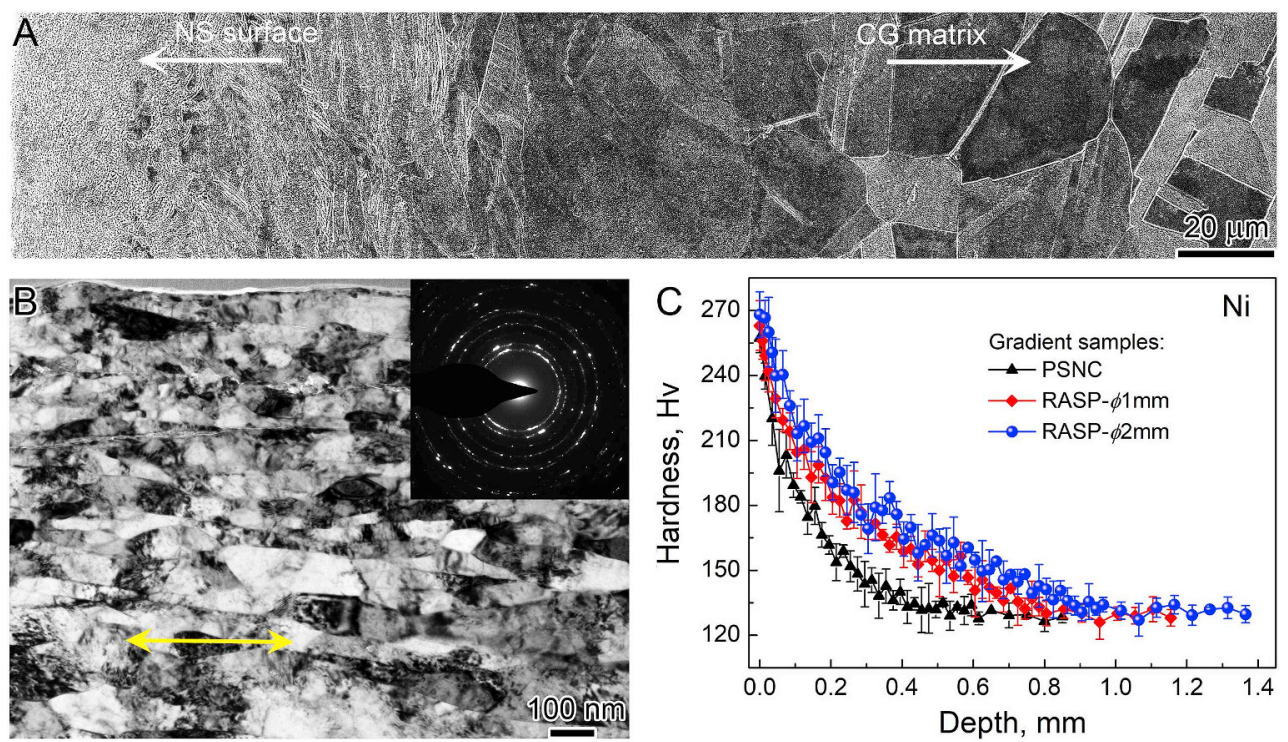

Fig. 3. (A) A gradient microstructure from nanostructured surface layer to coarse-grained central layer; $(B)$ Bright-field TEM image showing the NS at the topmost surface. $(C)$ Micro-hardness profiles measured in the mechanical gradient layer of as-received gradient samples. The double-arrowed yellow line in $(B)$ indicates the direction parallel to surface. (For interpretation of the references to color in this figure legend, the reader is referred to the Web version of this article.)

roughness contour for $\mathrm{Ni}_{\text {RASP- } \phi 2}, \mathrm{Ni}_{\mathrm{RASP}-\phi 1}$ and $\mathrm{Ni}_{\text {PSNC }}$ samples are measured as $80.8 \mu \mathrm{m}, 37.1 \mu \mathrm{m}$ and $2.1 \mu \mathrm{m}$, respectively. As shown, the sample processed with larger balls has higher roughness. The PSNC produced a very smooth surface due to its high striking frequency.

\subsection{Gradient microstructure and microhardness}

The $\mathrm{Ni}_{\text {RASP- } \phi 1}$ sample was used to show typical gradient microstructure. Fig. $3 A$ is a representative SEM image from the top NS layer to the CG central layer, which reveals a severely deformed sub-surface layer with obliterated initial grain boundary. Fig. $3 B$ is a typical TEM image taken at the topmost surface layer, showing a mixture of elongated and equiaxed nanostructures with high density of dislocations. The solid symbols in Fig. $3 C$ mark the microhardness profiles of the three types of gradient samples before tensile testing. As shown, the topmost layers of all samples have a hardness of $\sim 270 \mathrm{Hv}$, which is about twice that of the CG matrix. The thicknesses of the gradient layers of the $\mathrm{Ni}_{\text {RASP }-\phi 2}, \mathrm{Ni}_{\mathrm{RASP}-\phi 1}$ and $\mathrm{Ni}_{\mathrm{PSNC}}$ samples measured from these hardness profiles are $\sim 900 \mu \mathrm{m}$, $\sim 780 \mu \mathrm{m}$ and $\sim 450 \mu \mathrm{m}$, respectively. The detailed gradient microstructure and formation mechanism of such gradient samples were reported in previous works (Liu et al., 2015; Wang et al., 2006).

\subsection{Strength-ductility synergy}

Tensile specimens with only the topmost 400 - $\mu$ m-thick layer and the central 2.0 -mm-thick core layer of $\mathrm{Ni}_{\mathrm{RASP}-\phi 1}$ material are referred to as freestanding nanostructured gradient surface layer (NGSL) and homogeneous CG core, respectively. Fig. 4 compares the

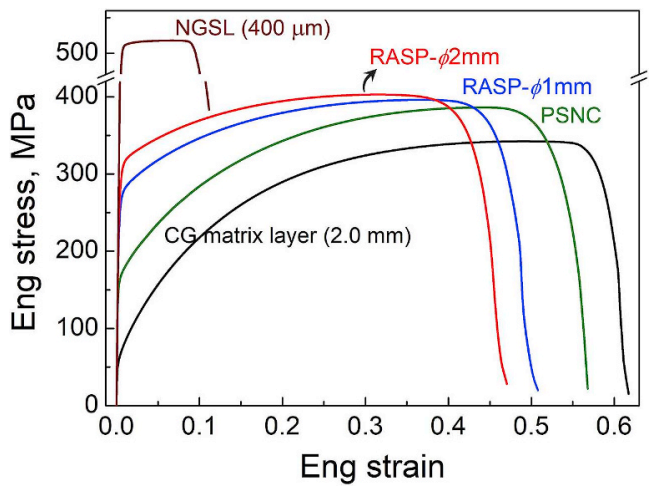

Fig. 4. Tensile engineering stress-strain curves for gradient samples and the freestanding NGSL and homogeneous CG matrix layer. 

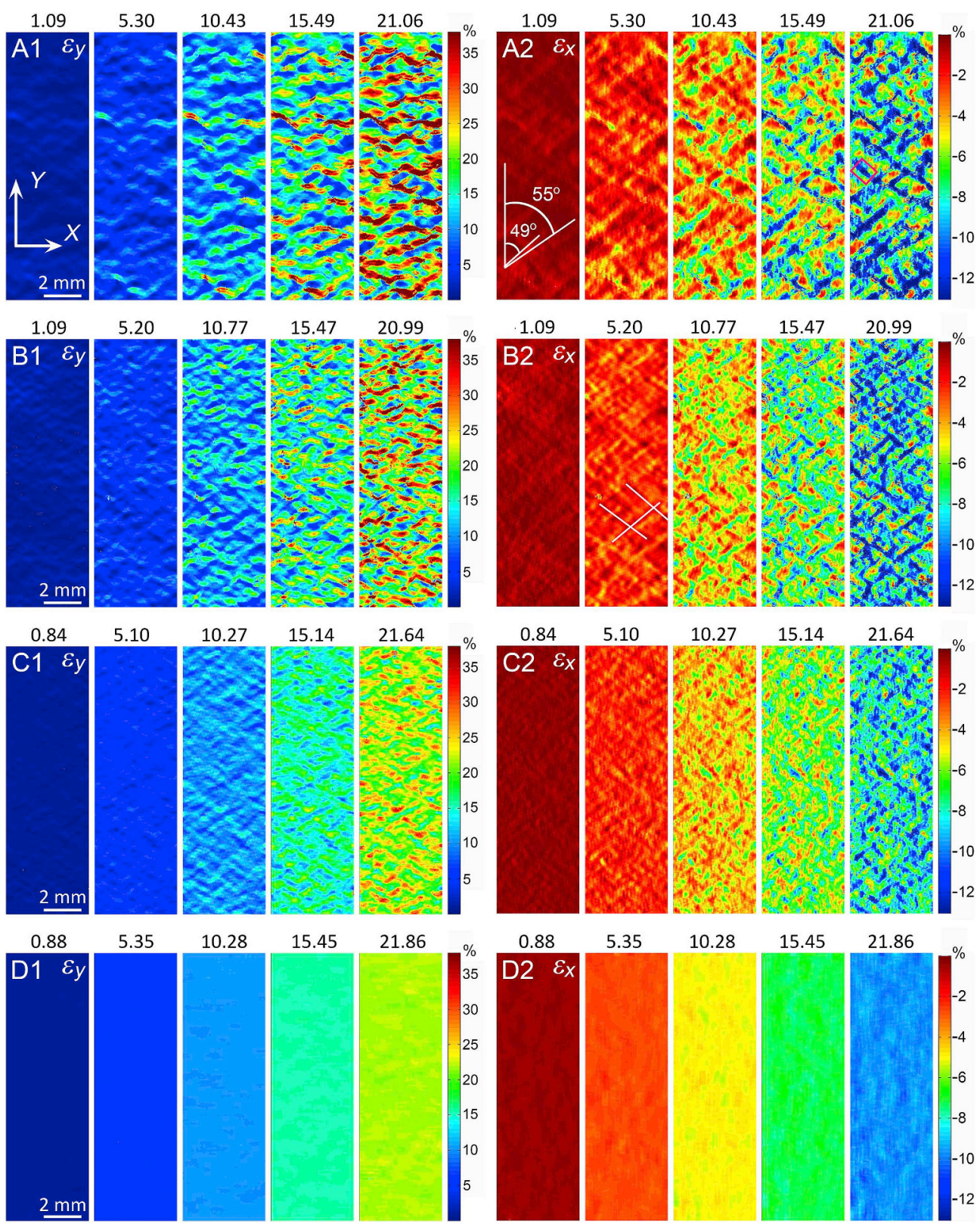

Fig. 5. The distributions of strain $\varepsilon_{y}$ (the left column) and corresponding $\varepsilon_{x}$ (the right column) on the surface of $\mathrm{Ni}_{\mathrm{RASP}-\phi 2}(A 1-A 2), \mathrm{Ni} \mathrm{RASP}_{\mathrm{R} 1}(B 1-B 2)$ and $\mathrm{Ni}_{\mathrm{PSNC}}(C 1-C 2)$ gradient samples and homogeneous CG (D1-D2) sample. They reveal the distribution and evolution of dispersive shear bands (SBs) in gradient sample. The shear bands are warm-colored in $\varepsilon_{y}$ contour and cold-colored in $\varepsilon_{x}$ contour. In the coordinate, $Y$ is the tensile loading direction and $X$ is the sample width direction. The number above each subgraph represents the average true tensile strain applied to the sample.

tensile behaviors of gradient samples, the freestanding NGSL and homogeneous CG layer. The gradient samples exhibit improved yield strength and excellent ductility (uniform elongation), which indicates a superior strength-ductility combination. Interestingly, the yield strength of the freestanding NGSL reaches $504 \pm 6 \mathrm{MPa}$, while its ductility is lowered to $\sim 6.8 \%$. These results indicate that the NS surface layers were well supported by the CG layer and did not fail prematurely.

\subsection{Dense dispersed shear bands in nanostructured layer}

Fig. 5A-C present the distribution and evolution of strains in the tensile direction $\varepsilon_{y}$ (the left five columns) and width direction $\varepsilon_{x}$ (the right five columns) on the nanostructured surface of gradient samples during uniform elongation. Surprisingly, dense macroscopic shear bands (SBs), orientated at $49^{\circ} \sim 55^{\circ}$ with respect to the tensile axis, are uniformly distributed over the whole surface of all 

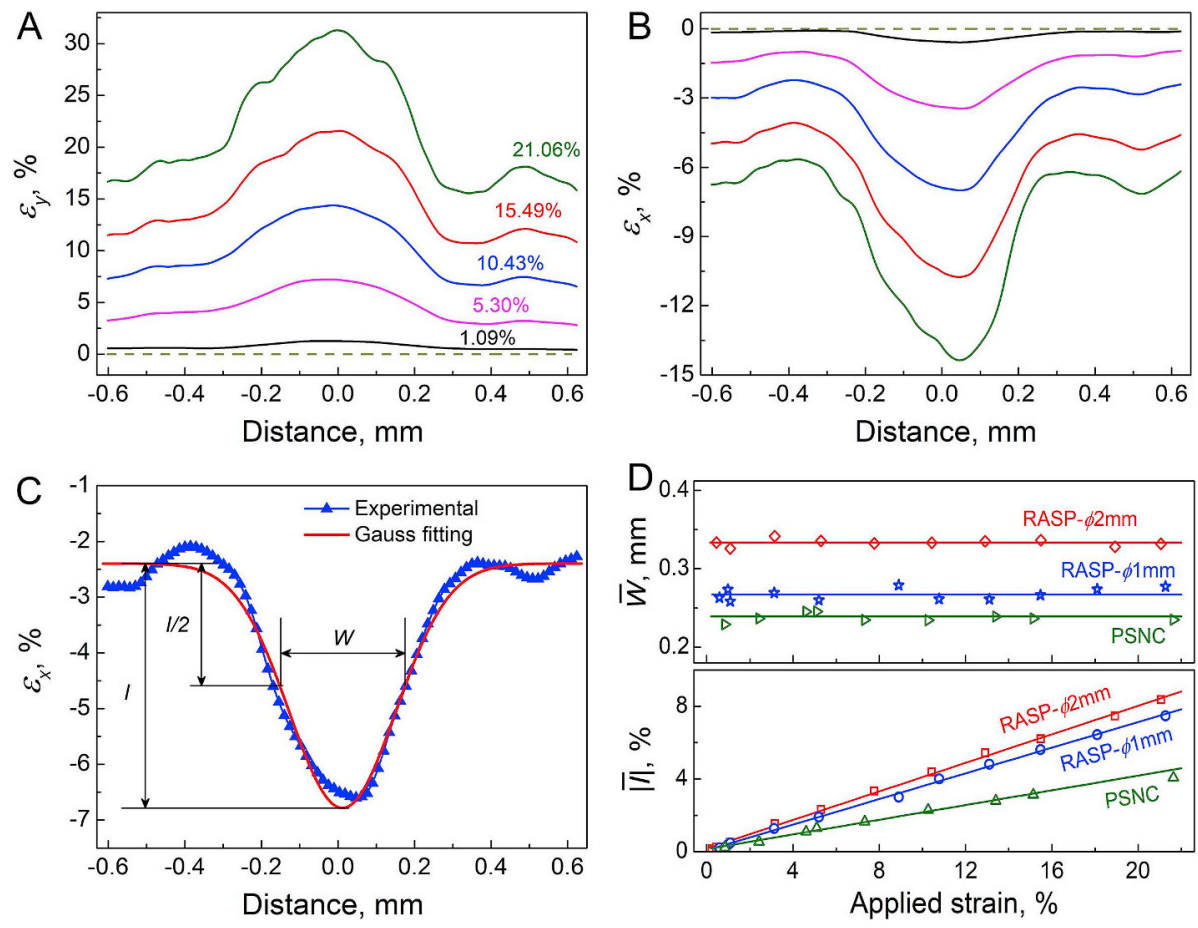

Fig. 6. Strain distribution and evolution in SBs. $(A)$ and $(B)$ are the statistically averaged distribution of $\varepsilon_{y}$ and $\varepsilon_{x}$ across a representative SBs in $\mathrm{Ni}_{\text {RASP- } \phi 2}$. Curves in $(A)$ and $(B)$ with the same color are strain distributions at the same strain state. $(C)$ Gauss fitting of the strain peak of a SB, from which the strain intensity $(I)$ and the full width at half maximum $(W)$ are measured. $(D)$ The evolution of $|\bar{I}|$ and $\bar{W}$ in the $\varepsilon_{x}$ peak of SBs in three types of gradient samples, with increasing applied strain. Each data point in $(D)$ was averaged from the values of 8 randomly selected SBs. (For interpretation of the references to color in this figure legend, the reader is referred to the Web version of this article.)

gradient samples. In contrast, such SBs are not found in the homogeneous CG sample (Fig. 5D), which implies that the low strain hardening capability of the NS layer is a precondition for the formation of SBs. It is also clear that the three types of gradient samples exhibit obvious difference in the morphology of SBs and the extent of strain inhomogeneity, which indicates significant effect of surface roughness and gradient layer thickness on the SB dimension, density and strain intensity.

For every type of gradient sample, the distribution and evolution of strains in 8 randomly selected SBs were analyzed. Taking a representative $\mathrm{SB}$ in the $\mathrm{Ni}_{\mathrm{RASP}-\phi 2}$ sample as an example, the distributions of $\varepsilon_{y}$ and $\varepsilon_{x}$ across the band at different tensile strains were statistically averaged along the shear banding direction and plotted in Fig. $6 A$ and $B$, respectively. As shown, the peak strain in SB is nearly twice that in the background (i.e. non-shear banding zone). It should be noted that the strains in both SBs and background increase with applied strain.

Fig. 6C demonstrates that the strain peak profile across a SB can be fitted with a Gauss function to extract two important parameters: the strain intensity $(I)$ and width at half maximum $(W)$. Fig. $6 D$ compares the evolutions of the average strain intensity $(\bar{I})$ and width at half maximum $(\bar{W})$ extracted from the $\varepsilon_{x}$ profile of the selected SBs, with increasing applied strain. As shown, the $\overline{|\bar{I}|}$ increased linearly with increasing applied strain, suggesting a stable plastic deformation in SBs. In addition, the $\bar{I} \mid$

Increased faster in the $\mathrm{Ni}_{\mathrm{RASP}-\phi 2}$ sample than in the $\mathrm{Ni}_{\mathrm{PSNC}}$ sample. The $\bar{W}$ remains largely constant during the whole plastic straining process, indicating constant SB widths, which contrasts the width growth in gradient IF steel (Yuan et al., 2019). The $\bar{W}$ of $\varepsilon_{x}$ strain peaks in the $\mathrm{Ni}_{\text {RASP- } \phi 2}, \mathrm{Ni}_{\text {RASP- } \phi 1}$ and $\mathrm{Ni}_{\text {PSNC }}$ samples are $\sim 0.33 \mathrm{~mm}, \sim 0.27 \mathrm{~mm}$ and $\sim 0.24 \mathrm{~mm}$, respectively. These values are much larger than those in homogeneous NS/UFG bulks (Jia et al., 2003, 2013; Hong et al., 2010; Carsley et al., 1995, 1998).

Fig. 7 shows $\mathrm{SB}$ nucleation at early strain stages in a $\mathrm{Ni}_{\mathrm{RASP}-\phi 2}$ sample. SBs started to nucleate at the elastic-plastic transition stage (indicated by the white arrows). The density evolution of SBs, total length of SBs per unit area, as a function of applied strain is shown in Fig. 8. It is revealed that for all samples the SBs density increased quickly at the elastic-plastic transition and low plastic-strain stages, and then reached saturation at $\sim 3 \%$ strain. It is also clear that the saturated density of SBs in the $\mathrm{Ni}_{\mathrm{PSNC}}$ sample with smoother surface is nearly double that in the $\mathrm{Ni}_{\mathrm{RASP}-\phi 2}$ sample.

Fig. 9 shows the SB evolution in a stand-alone NGSL. As indicate by the white arrow, a dominant SB with extremely high strain concentration quickly developed, penetrated through the cross-section and then caused fracture, leading to low ductility. Meanwhile, the SBs in uniform gauge section exhibit a lower density than in the integrated $\mathrm{Ni}_{\mathrm{RASP}-\phi 1}$ sample (Fig. $5 B$ ). These results suggest that dense dispersive SBs can be activated only when NS surface layers and CG matrix are deformed together. 

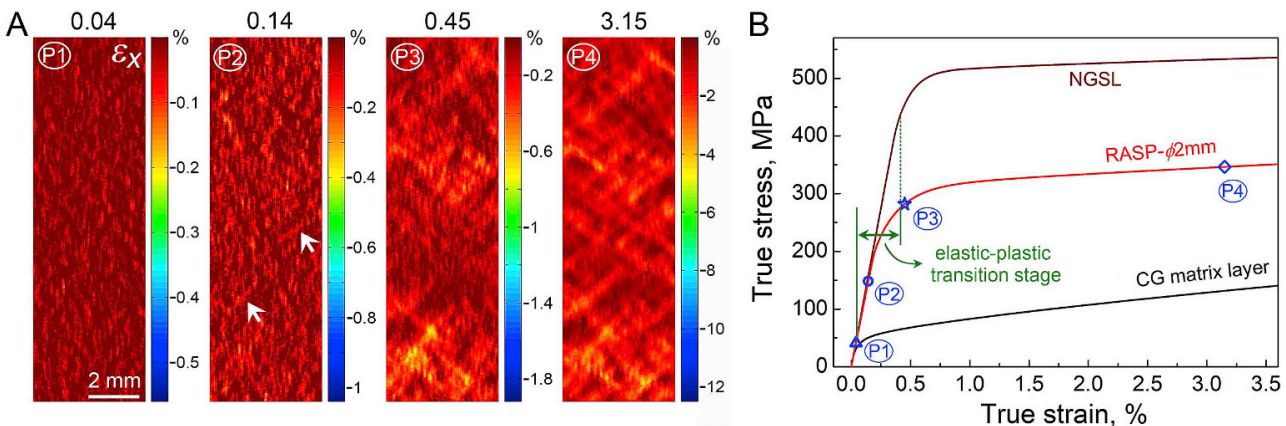

Fig. 7. (A) SBs on the surface of $\mathrm{Ni}_{\mathrm{RASP}-\phi 2}$ sample at different strain stages, revealing the fast increase of SBs density in elastic-plastic transition stage (P2-P3) and low plastic-strain stage (P3-P4). The white arrows indicate the initial embryos of inclined strain bands. (B) True stress-strain curves, showing the strain stage of P1-P4.

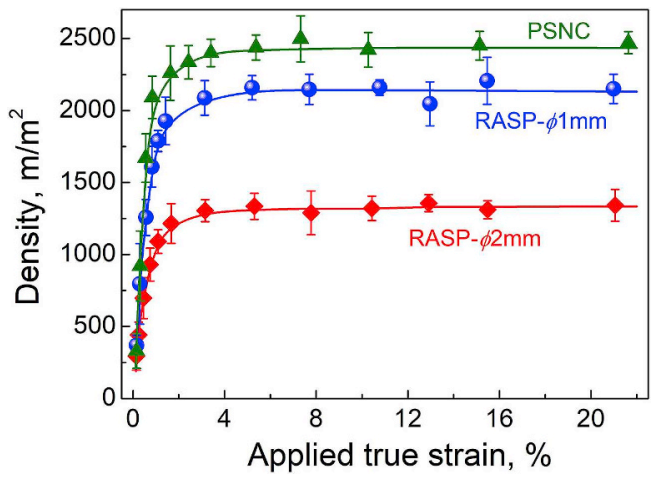

Fig. 8. The density evolution of SBs in gradient samples.
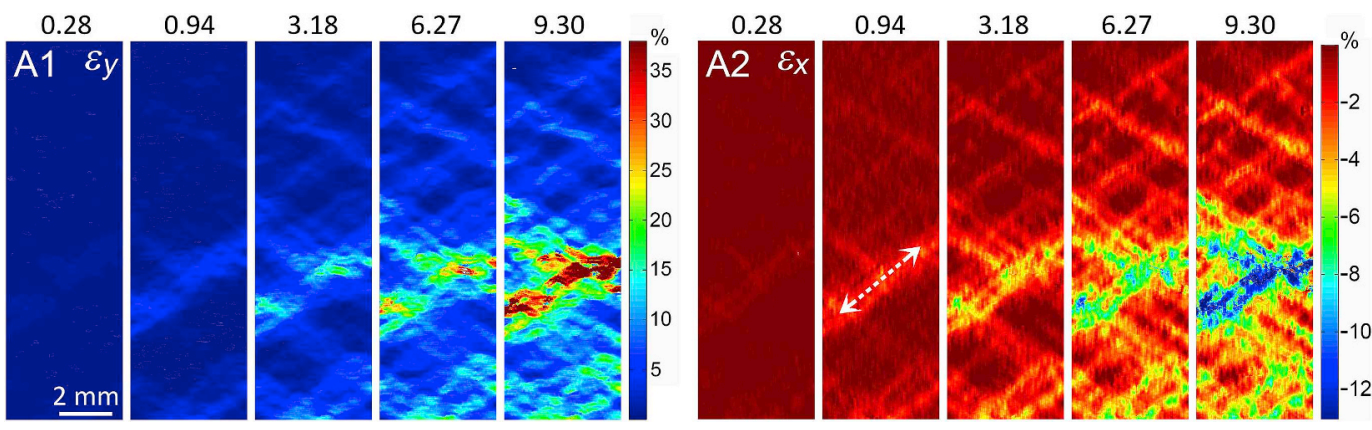

Fig. 9. SBs on the surface of freestanding NGSL that peeled from $\mathrm{Ni}_{\mathrm{RASP}-\phi 1}$ material: (A1) $\varepsilon_{y}$; (A2) $\varepsilon_{x}$. The double-headed arrow indicates the runaway SB.

\section{Discussion}

The experimental results show that the enhanced plasticity of NS surface layer in gradient structure can be primarily attributed to the formation of dense macroscopic SBs and their stable evolution. Such dispersed and stable shear banding deformation was never observed in homogeneous metals under tension, which suggests that this mechanism is unique to the NS-CG gradient structure.

\subsection{Unique characteristics of dispersed shear bands}

As a non-crystallographic deformation mode, SBs are also often observed in materials without enough strain hardening to maintain uniform plastic flow, such as NS/UFG bulk metals and metallic glasses (Yang et al., 2010; Cheng et al., 2005; Hong et al., 2010; Jia et al., 2003; Carsley et al., 1995, 1998; Lu et al., 2013; Hays et al., 2000). It is interesting that both the configuration and evolution of the SBs observed here are very different from those in conventional homogeneous materials.

First, the SBs in gradient $\mathrm{Ni}$ are observed to distribute uniformly over the whole gauge section, and the plastic deformation in the SBs evolved stably with increasing applied strain (Fig. 6D). In contrast, in NS/UFG metals, SBs propagate quickly to fail the sample 


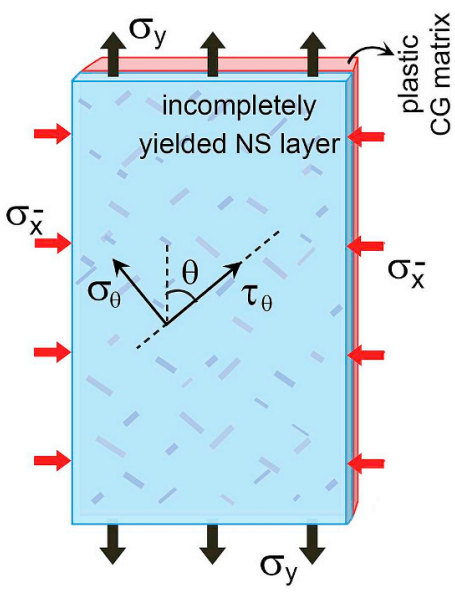

Fig. 10. Schematic illustration of the load and constraint applied in the NS surface layer of gradient sample at the elastic-plastic transition stage. $\sigma_{y}$ is external load, and $\sigma_{x}^{-}$is the compressive constraint from plastic CG matrix. $\theta$ is the preferential shear banding plane. The tilted short bands indicate the formation of early SBs.

due to the low strain hardening capacity and lack of constraint, and the gauge region far away from neck or fracture zone generally exhibits no SBs (Cheng et al., 2005; Yang et al., 2010). Second, the SBs in the gradient sample have macroscopic sizes in the order of millimeters (Figs. 6 and $5(A-C)$ ). In contrast, except for the predominant unstable SB, the height of most SBs in the NS/UFG bulks is in the range of several tens or hundreds of nanometers, and their width generally ranges from submicron to several microns, i.e., micro SBs (Jia et al., 2003; Lu et al., 2013; Yang et al., 2010). Third, the density of SBs in gradient Ni reached saturation at $\sim 3 \%$ strain (Fig. 8), while new SBs and branches in NS materials are nucleated through the whole plastic straining process (Jia et al., 2003). Fourth, the constant width of SBs in the gradient Ni differs greatly from the continuous thickening behavior of micro SBs in NS metals (Carsley et al., 1995; Hong et al., 2010; Jia et al., 2003). This is also different from the earlier report of SB widening in gradient IF steel (Yuan et al., 2019).

\subsection{Nucleation of dispersed shear bands}

SBs may be nucleated when the local shear stress $\left(\tau_{\theta}\right)$ on the preferential shear banding plane $(\theta)$ reaches the critical value for shear instability $\left(\tau_{c \theta}\right)$, which is preferentially activated at stress concentration sites under load (Ardeljan et al., 2015; He et al., 2003; Jia et al., 2013). In heterostructured materials, the boundaries between soft and hard domains are likely sites for stress concentration due to the mutual constraint-induced complex stress state and dislocation pile-up (C. X. Huang et al., 2018; M. Huang et al., 2018; Wang et al., 2019c; Wu et al., 2015).

For the gradient structure, during the elastic-plastic transition stage (see Fig. 7B), the elastic NS surface layer and the plastic inner layers form an elastic-plastic domain boundary. Due to the incompatibility in lateral shrinking rate between the inner plastic CG (apparent Poisson ratio $\nu \approx 0.5)$ and the elastic NS surface layer $(\nu \approx 0.33)$, a lateral compression stress $\left(\sigma_{x}^{-}\right)$is added to the NS surface layer (see Fig. 10) (Wang et al., 2018b; Wu et al., 2014b). This increases the shear stress $\tau_{\theta}$ from $\sigma_{y} \sin \theta \cos \theta$ to $\left(\sigma_{y}+\left|\sigma_{x}^{-}\right|\right) \sin \theta \cos \theta$. In addition, the dislocation pile-up near this elastic/plastic interface also contributes to stress concentration (Wu et al., 2015; Zeng et al., 2016). As verified by the Vickers hardness map in Fig. 11, the surface layers themselves exhibit significant hardness variation, which indicates the existence of in-layer soft/hard domain boundary. This is expected to introduce stress/strain inhomogeneity and concentration sites as well (Sun et al., 2009; Wu and Zhu, 2017). Hence, the NS surface layers in gradient materials are believed to have abundant dispersed potential sites for nucleating SBs.

During deformation, once the stress concentration is built high enough (reaches $\tau_{c \theta}$ ) to induce plastic deformation across a domain boundary, the local plastic deformation may become unstable in the NS surface layer because the harder domain likely has finer grain size and low strain hardening capability (Ardeljan et al., 2015; Wang et al., 2019b). This will nucleate a local SB, whose propagation in the length direction can only be stopped by another hard domain with even higher strength in the NS layer. This effect is similar to the activation of SBs in NS Ti composite (He et al., 2003), bimodal structured Cu (Wang et al., 2019b), and heterophase laminate (Jia et al., 2013), where the SB nucleation was promoted by the interaction-induced high internal stress at domain boundaries.

As discussed above, SBs can be effectively arrested by the neighboring harder domains in the NS layer due to the strength inhomogeneity. In the depth direction, a propagating SB will meet layers with larger grains whose higher plasticity will lower the stress at the band front, while their stronger work hardening helps with arresting the propagation. The arrest of propagating SBs provides opportunity to nucleate more SBs in less optimal regions, which eventually leads to the nucleation of high density of dispersed SBs that are homogeneously distributed over the whole NS layer. This is the primary mechanism for the fast increase of SB density at the yielding and low plastic-strain stages (Figs. 7 and 8). This process is similar to the arrestment of propagating SBs and the activation of multiple SBs in inhomogeneous metallic glasses consisting of ductile phase or layers (Hays et al., 2000; Kosiba et al., 2019; Lu et al., 2013; Sha et al., 2017; Zhou et al., 2013). 


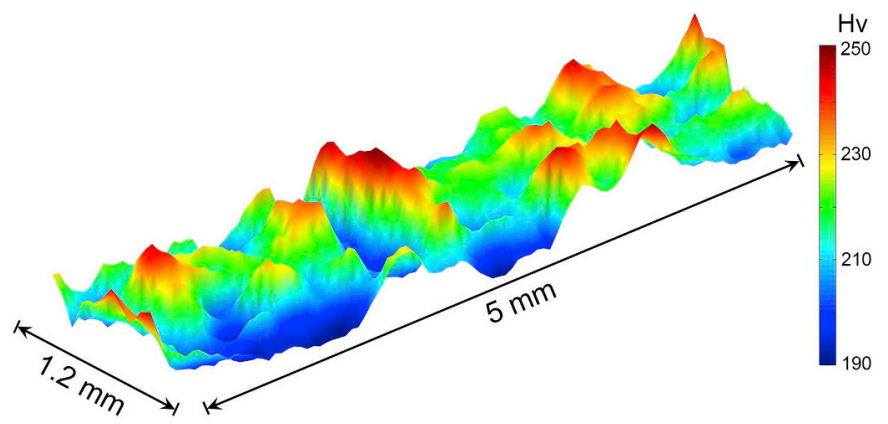

Fig. 11. Vickers hardness contour measured on the sub-surface layer (at the depth of $130 \mu \mathrm{m}$ ) of as-received $\mathrm{Ni}_{\mathrm{RASP}-\phi 2}$ sample. The data map contains the value of 2400 homogeneously distributed indentations. The indent depth is $\sim 2.75-3.15 \mu \mathrm{m}$.

The orientation of SBs observed here is not along the plane with maximum shear stress, i.e., $45^{\circ}$ with respect to the loading axis. This is a glasslike local shear instability response (Carsley et al., 1995; Donovan, 1989; Zhang et al., 2003), which has also been observed in high-strength NS and UFG metals. For example, micro SBs in UFG Pd-Ag alloy oriented $\sim 50^{\circ}$ to the tensile direction (Yang et al., 2010). Asymmetric shear banding orientations under tension and compression were observed in NS Fe-Cu alloy (Carsley et al., 1998, 1995). It has been proposed that such behaviors can be attributed to the yielding-related shear banding process and the normal stress-dependent yielding mechanism (Carsley et al., 1998). As described in the Mohr-Coulomb criterion $\left(\tau_{y \theta}=\tau_{0}-\mu \sigma_{\theta}\right)$, the shear yielding stress $\left(\tau_{y \theta}\right)$ on inclined $\theta$ plane of NS metals is sensitive to the in-plane normal stress $\left(\sigma_{\theta}\right)$ (Donovan, 1989; Zhang et al., 2003). For the NS surface layer of gradient Ni sample under tension, the $\tau_{y \theta}$ is met more easily on planes $49^{\circ} \sim 55^{\circ}$ to the tensile axis, which induces plastic shear banding in this angle range.

\subsection{Stable evolution of dispersed shear bands}

As demonstrated by the linear evolution of strain intensity in SBs (Fig. 6D), SBs in NS layer remain stable to very large applied tensile strains without serious strain localization to fracture the sample.

Without the CG central layer the freestanding NGSL formed a dominant SB that caused the early fracture (Fig. 9), which verifies the critical role of the CG central layer in stabilizing SBs propagation. Fig. 12 presents the DIC strain mappings characterized on the lateral surface of gradient sample. As shown, SBs were originated from NS surface layers and weakened along depth with a gradual decrease in strain intensity. They partially intersected with each other, and were terminated by CG interior at the depth of $\sim 650 \mu \mathrm{m}$. On the other hand, the in-layer mutual intersection of SBs constrained their in-layer propagation (Fig. $5 A$ - $C$ ). These processes blunted bands sharpness, and hindered their catastrophic development, leading to a stable propagation process. Stabilization of strain localization by the neighboring softer domain or incompatible interface has also been reported in other heterogeneous structures (Azizi et al., 2018; Chen et al., 2008; Wu et al., 2017; Zhu and Lu, 2012).

The CG interior of present gradient Ni sample may also play a role in constraining the widening of dispersed SBs. In this study, the SB width remained constant with increasing strain (Fig. 6D), which is very different from the sustained widening of SB observed in the NS layer of gradient IF steel (Yuan et al., 2019). A closer examination of the gradient IF steel sample found that the gradient layer extended all the way to the center due to thin whole sample thickness $(1 \mathrm{~mm})$. In contrast, the Ni sample used here is much thicker $(3.6 \mathrm{~mm})$, which resulted in a much thicker CG central layer. Therefore, the likely reason for this difference is that the CG core in the gradient IF steel is too thin to effectively constrain the widening of SBs.

Moreover, the strain intensity of SBs increased gradually with applied strain (Fig. 6), which indicates that large strain gradient was formed in the shear banding zone during straining. As reported in gradient structured IF steel and CG/NS laminates, such strain gradient increase is known to lead to the accumulation of geometrically necessary dislocations and the development of back-stress work hardening (C. X. Huang et al., 2018; Li et al., 2017; Yang et al., 2016). The enhanced strain hardening will help to stabilize the plastic flow in SBs.

\subsection{Microstructure evolution in shear bands}

Dispersed SBs stabilize the uniform elongation of NS surface layer, which verifies a long-term conjecture that NS metals may serve as ductile materials as long as catastrophic strain localization is effectively suppressed (Fang et al., 2011; Yuan et al., 2019). An issue arising here is how the microstructure in SBs evolves to accommodate such large tensile strain.

We examined the microstructure of the topmost NS layers of $\mathrm{Ni}_{\mathrm{RASP}-\phi 1}$ sample before and after tension (Fig. 13). The microstructure of as-processed surface layer is characterized by largely elongated grains with an average transversal grain size of $68 \mathrm{~nm}$ and an aspect ratio of 4.17 (Fig. 13A1 and A2). After deformation, surprisingly, equiaxed grains with even larger size and lower dislocation density are developed in shear banding zone (Fig. 13B1 and B2). These observations suggest that the plastic deformation of shear banding zone is dominated by grain coarsening, which has been interpreted as a mechanically-driven grain boundary migration 


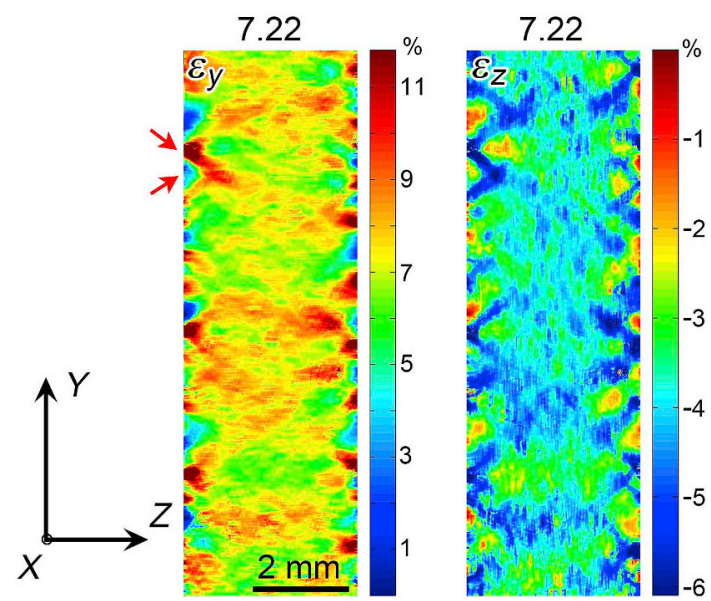

Fig. 12. SBs on the lateral surface of $\mathrm{Ni}_{\mathrm{RASP}-\phi 1}$ sample, measured at the applied strain of $7.22 \%$. $Z$ represents the thickness direction with microstructure gradient.

mechanism in NS metals (Chen et al., 2017; Fang et al., 2011; Wang et al., 2019a). The increase of grain size and change of aspect ratio are also detected in the zones outside SBs (Fig. $13 \mathrm{C1}$ and C2). However, comparing to that in shear banding zone, the extent of grain coarsening is less significant and the initial NS remains in local regions (marked by dotted cycles), suggesting that the rate and extent of grain boundary migration are positively correlated with stress/strain concentrations (Rupert et al., 2009).

As marked by red arrows in Fig. 13C1, under stress grain boundary migration occurred at the expense of neighboring highlydefective microstructures. This procedure results in dismantlement and annihilation of initial defects, thereby accommodating applied strain. The migration of grain boundary can be interpreted as a releasing process of distortion energy (Legros et al., 2008; Wang et al., 2012). Accompanying grain boundary migration in SBs, the formation of equiaxed grains with relatively low dislocation density provides substantial available room for defects storage (Fig. 13B1), which implies the regaining of strain hardening capability for shear banding zone (Huang et al., 2015; Yuan et al., 2019). With further increase in applied strain, the dominant plastic mechanism in shear banding zone will shift from grain boundary migration to conventional dislocation slip as the size of grown grains tends to saturate (Fang et al., 2011). This is the reason why some equiaxed coarse grains in shear banding zone exhibit high dislocation density (Fig. 13B1). More importantly, the regained strain hardening capability may also play a crucial role in stabilizing shear banding deformation.

\subsection{Effects of surface roughness and strength heterogeneity on shear banding}

Surface roughness could significantly affect the morphology of SBs, but is not the primary reason for SB nucleation. As shown in Fig. 2, the PSNC sample had a very smooth surface, but dispersed SBs appeared in its NS layer (Fig. 5C) with higher density than in the RASP samples which had much higher surface roughness (Fig. 8).

The statistical structural parameters of gradient samples, and the geometrical parameters and strain intensity of SBs are summarized in Table 2. It can be seen that SBs in samples with bigger surface roughness exhibit greater width, higher strain intensity but lower spatial density. This can be attributed to the effect of concave-convex roughness pattern on the development of stress concentration. Bigger peening balls produced the wider and deeper valleys on the surface layer (Table 2 and Fig. 2), which consequently led to larger stress concentration sites with longer inter-site distance and higher stress concentration under load.

Since the NS surface layers in all of the gradient samples were produced by the impact of high-speed balls or indenter, it was inevitable for the deformation to be heterogeneous (Panin et al., 2015). This led to inhomogeneous distribution of hardness (strength) in surface layers (Fig. 11), and thus provided extra soft/hard incompatible boundaries for stress concentration and strength heterogeneity to arrest the propagation of early SBs during deformation.

\section{Conclusions}

In summary, a unique plastic strain accommodation mechanism, formation of dense dispersed SBs, was experimentally revealed in gradient-structured Ni using DIC strain measurements. The main conclusions can be drawn as follow.

(1) Dense dispersed SBs were uniformly distributed over the NS surface layer, and evolved stably during the entire plastic deformation, resulting in excellent uniform elongations (larger than 30.6\%) which were much larger than that of a freestanding NS surface layer $(\sim 6.8 \%)$.

(2) The nucleation of dispersed SBs started at an early elastic-plastic transition stage, and reached saturation at $\sim 3 \%$ strain. The gradient microstructure, surface roughness and hardness variation introduced abundant soft/hard domain boundaries in the NS 

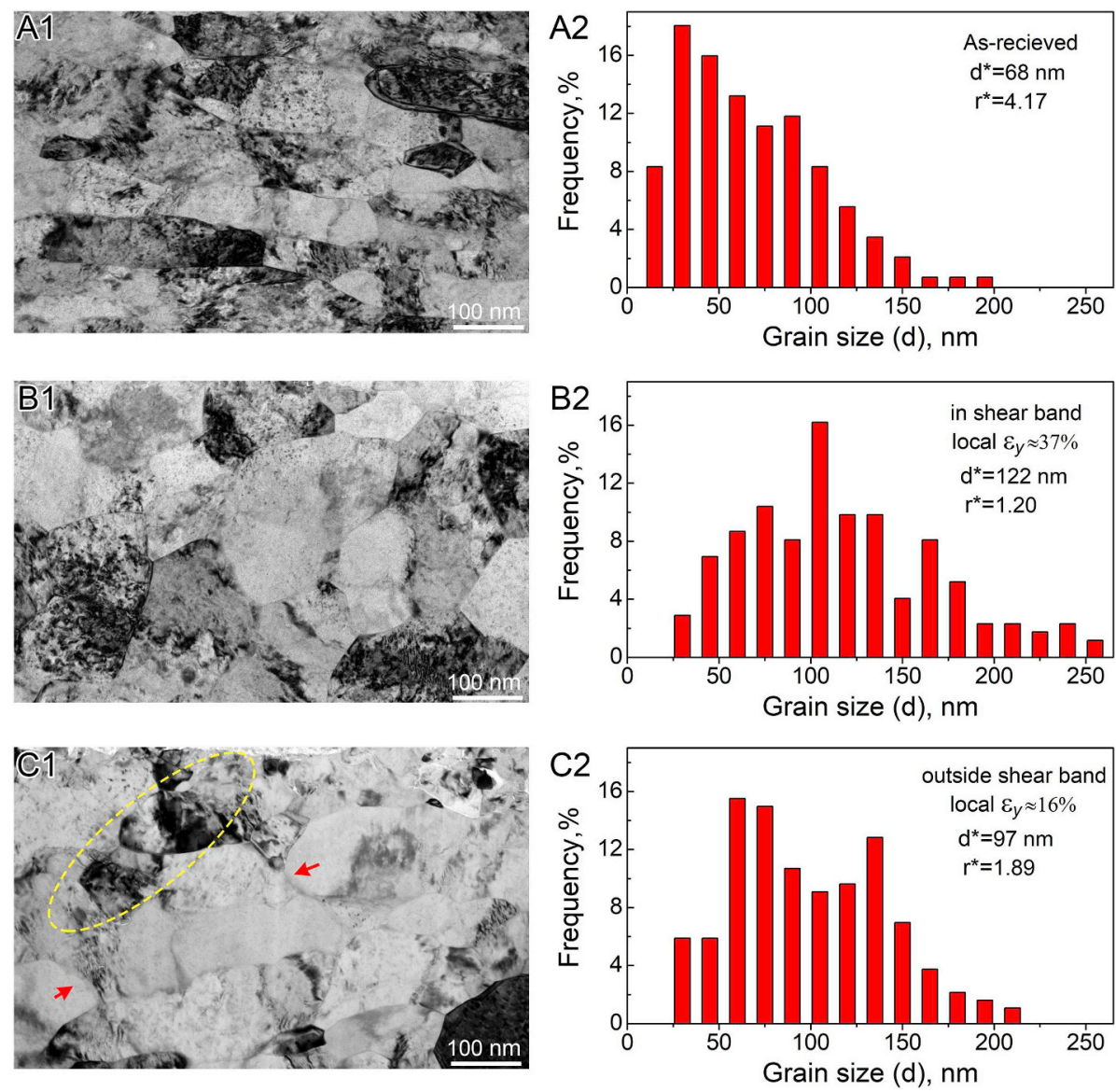

Fig. 13. Bright-filed TEM micrographs and the statistical distribution of grain size in the topmost layer of $\mathrm{Ni}_{\mathrm{RASP}-\phi 1}:(A 1, A 2) \mathrm{As}-\mathrm{received}$; $(B 1, B 2)$ shear banding zone with a local $\varepsilon_{y}$ of $\sim 37 \%$; $(C 1, C 2)$ non-shear banding matrix with a local $\varepsilon_{y}$ of $\sim 16 \%$. TEM foils of $(B)$ and $(C)$ were extracted from the SB and the neighboring matrix indicated by red arrows in Fig. 12, at the applied strain (whole-field average) of $21.7 \%$. Dotted cycles and red arrows in (C1) mark the residue initial NS and the migrating grain boundary, respectively. (For interpretation of the references to color in this figure legend, the reader is referred to the Web version of this article.)

Table 2

The structural parameters of gradient samples and the geometrical parameters of SBs, including the maximum height difference between the convex peak and concave valley $(H)$ and the average space between neighboring concave valleys $\left(\overline{d_{v}}\right)$ of surface roughness contour, the thickness of gradient layer $\left(t_{G}\right)$, the thickness ratio of gradient layer to sample $\left(t_{G} / t_{S}\right)$, the saturated SB density $\left(\rho_{b}\right)$, the average space between neighboring SBs $\left(\overline{d_{b}}\right)$, the average strain intensity $(\bar{I} \mid)$ and width at half intensity $(\bar{W})$ of $\varepsilon_{x}$ peaks at the tensile strain of $\sim 15.5 \%$.

\begin{tabular}{|c|c|c|c|c|c|c|c|c|}
\hline \multirow[t]{2}{*}{ Material } & \multicolumn{2}{|c|}{ Roughness } & \multicolumn{2}{|c|}{ Gradient layer } & \multicolumn{4}{|l|}{ SBs } \\
\hline & $H, \mu \mathrm{m}$ & $\overline{d_{v}}, \mathrm{~mm}$ & $t_{G}, \mu \mathrm{m}$ & $t_{G} / t_{I}$ & $\rho_{b}, \mathrm{~m} / \mathrm{m}^{2}$ & $\overline{d_{b}}, \mathrm{~mm}$ & $\bar{W}, \mathrm{~mm}$ & $\overline{|I|}, \%$ \\
\hline $\mathrm{Ni}_{\text {RASP }-\phi 2}$ & 80.8 & $0.82 \pm 0.46$ & 900 & 0.5 & $1330 \pm 100$ & $0.91 \pm 0.67$ & $0.33 \pm 0.12$ & $6.20 \pm 3.8$ \\
\hline $\mathrm{Ni}_{\mathrm{RASP}-\phi 1}$ & 37.1 & $0.38 \pm 0.23$ & 780 & 0.43 & $2140 \pm 150$ & $0.56 \pm 0.44$ & $0.27 \pm 0.09$ & $5.62 \pm 4.0$ \\
\hline $\mathrm{Ni}_{\text {PSNC }}$ & 2.1 & - & 450 & 0.25 & $2430 \pm 120$ & $0.49 \pm 0.31$ & $0.24 \pm 0.11$ & $3.13 \pm 2.1$ \\
\hline
\end{tabular}

surface layer. The stress concentration induced by elastic/plastic interaction at domain boundaries promoted the early nucleation of SBs. The CG matrix and neighboring hard domains arrested the propagation of early SBs, which provided an opportunity to nucleate more SBs at dispersed stress concentration sites.

(3) Stable shear banding at the large strain stage was maintained by the stabilization and mechanical constraints from the central CG matrix and the mutual intersection of SBs.

(4) The large applied strain in shear bands was accommodated by mechanically-driven grain boundary migration. Grain coarsening in shear bands led to the regaining of strain hardening capability, which helps to stabilize shear banding deformation.

(5) Surface roughness affected the morphology of SBs by affecting the stress concentration in the surface layer. 


\section{Acknowledgements}

This work was supported by the National Key R\&D Program of China (2017YFA0204403), the National Natural Science Foundation of China (Nos.11672195, 51931003 and 51741106) and Sichuan Youth Science and Technology Foundation (2016JQ0047). Yanfei Wang would like to acknowledge the support from Chinese Scholar Council.

\section{References}

Ardeljan, M., Knezevic, M., Nizolek, T., Beyerlein, I.J., Mara, N.A., Pollock, T.M., 2015. A study of microstructure-driven strain localizations in two-phase polycrystalline HCP/BCC composites using a multi-scale model. Int. J. Plast. 74, 35-57. https://doi.org/10.1016/j.ijplas.2015.06.003.

Asaro, R.J., 1983. Micromechanics of crystals and polycrystals. Elsevier. Adv. Appl. Mech. 1-115. https://doi.org/10.1016/S0065-2156(08)70242-4.

Azizi, H., Zurob, H.S., Embury, D., Wang, X., Wang, K., Bose, B., 2018. Using architectured materials to control localized shear fracture. Acta Mater. 143, 298-305. https://doi.org/10.1016/j.actamat.2017.10.027.

Borg, U., 2007. Strain gradient crystal plasticity effects on flow localization. Int. J. Plast. 23, 1400-1416. https://doi.org/10.1016/j.ijplas.2007.01.003.

Carsley, J.E., Fisher, A., Milligan, W.W., Aifantis, E.C., 1998. Mechanical behavior of a bulk nanostructured iron alloy. Metall. Mater. Trans. A 29, 2261-2271. https:// doi.org/10.1007/s11661-998-0104-3.

Carsley, J.E., Milligan, W.W., Hackney, S.A., Aifantis, E.C., 1995. Glasslike behavior in a nanostructured Fe/Cu alloy. Metall. Mater. Trans. A 26, 2479-2481. https:// doi.org/10.1007/BF02671262.

Chen, A., Li, D., Zhang, J., Song, H., Lu, J., 2008. Make nanostructured metal exceptionally tough by introducing non-localized fracture behaviors. Scr. Mater. 59, 579-582. https://doi.org/10.1016/j.scriptamat.2008.04.048.

Chen, W., You, Z.S., Tao, N.R., Jin, Z.H., Lu, L., 2017. Mechanically-induced grain coarsening in gradient nano-grained copper. Acta Mater. 125, 255-264. https://doi. org/10.1016/j.actamat.2016.12.006.

Cheng, S., Ma, E., Wang, Y., Kecskes, L., Youssef, K., Koch, C., Trociewitz, U., Han, K., 2005. Tensile properties of in situ consolidated nanocrystalline Cu. Acta Mater. 53, 1521-1533. https://doi.org/10.1016/j.actamat.2004.12.005.

Cheng, Z., Zhou, H.F., Lu, Q.H., Gao, H.J., Lu, L., 2018. Extra strengthening and work hardening in gradient nanotwinned metals. Science 362, eaau1925. https://doi. org/10.1126/science.aau1925.

Donovan, P.E., 1989. A yield criterion for Pd40Ni40P20 metallic glass. Acta Metall. 37, 445-456. https://doi.org/10.1016/0001-6160(89)90228-9.

Fang, T.H., Li, W.L., Tao, N.R., Lu, K., 2011. Revealing extraordinary intrinsic tensile plasticity in gradient nano-grained copper. Science 331, 1587-1590. https://doi. org/10.1126/science.1200177.

Hays, C.C., Kim, C.P., Johnson, W.L., 2000. Microstructure controlled shear band pattern formation and enhanced plasticity of bulk metallic glasses containing in situ formed ductile phase dendrite dispersions. Phys. Rev. Lett. 84, 2901-2904. https://doi.org/10.1103/PhysRevLett.84.2901.

He, G., Eckert, J., Löser, W., Schultz, L., 2003. Novel Ti-base nanostructure-dendrite composite with enhanced plasticity. Nat. Mater. 2, 33-37. https://doi.org/10. 1038/nmat792.

Hong, C.S., Tao, N.R., Huang, X., Lu, K., 2010. Nucleation and thickening of shear bands in nano-scale twin/matrix lamellae of a Cu-Al alloy processed by dynamic plastic deformation. Acta Mater. 58, 3103-3116. https://doi.org/10.1016/j.actamat.2010.01.049.

Huang, C.X., Hu, W.P., Wang, Q.Y., Wang, C., Yang, G., Zhu, Y.T., 2015. An ideal ultrafine-grained structure for high strength and high ductility. Mater. Res. Lett. 3, 88-94. https://doi.org/10.1080/21663831.2014.968680.

Huang, C.X., Wang, Y.F., Ma, X.L., Yin, S., Höppel, H.W., Göken, M., Wu, X.L., Gao, H.J., Zhu, Y.T., 2018. Interface affected zone for optimal strength and ductility in heterogeneous laminate. Mater. Today 21, 713-719. https://doi.org/10.1016/j.mattod.2018.03.006.

Huang, M., Xu, C., Fan, G.H., Maawad, E., Gan, W.M., Geng, L., Lin, F.X., Tang, G.Z., Wu, H., Du, Y., Li, D.Y., Miao, K.S., Zhang, T.T., Yang, X.S., Xia, Y.P., Cao, G.J., Kang, H.J., Wang, T.M., Xiao, T.Q., Xie, H.L., 2018. Role of layered structure in ductility improvement of layered Ti-Al metal composite. Acta Mater. 153, 235-249. https://doi.org/10.1016/j.actamat.2018.05.005.

Jia, D., Ramesh, K.T., Ma, E., 2003. Effects of nanocrystalline and ultrafine grain sizes on constitutive behavior and shear bands in iron. Acta Mater. 51, 3495-3509. https://doi.org/10.1016/S1359-6454(03)00169-1.

Jia, N., Roters, F., Eisenlohr, P., Kords, C., Raabe, D., 2012. Non-crystallographic shear banding in crystal plasticity FEM simulations: example of texture evolution in $\alpha$ brass. Acta Mater. 60, 1099-1115. https://doi.org/10.1016/j.actamat.2011.10.047.

Jia, N., Roters, F., Eisenlohr, P., Raabe, D., Zhao, X., 2013. Simulation of shear banding in heterophase co-deformation: example of plane strain compressed Cu-Ag and Cu-Nb metal matrix composites. Acta Mater. 61, 4591-4606. https://doi.org/10.1016/j.actamat.2013.04.029.

Kassner, M.E., Geantil, P., Levine, L.E., 2013. Long range internal stresses in single-phase crystalline materials. Int. J. Plast. 45, 44-60. https://doi.org/10.1016/j. ijplas.2012.10.003.

Kosiba, K., Şopu, D., Scudino, S., Zhang, L., Bednarcik, J., Pauly, S., 2019. Modulating heterogeneity and plasticity in bulk metallic glasses: role of interfaces on shear banding. Int. J. Plast. 119, 156-170. https://doi.org/10.1016/j.ijplas.2019.03.007.

Legros, M., Gianola, D.S., Hemker, K.J., 2008. In situ TEM observations of fast grain-boundary motion in stressed nanocrystalline aluminum films. Acta Mater. 56, 3380-3393. https://doi.org/10.1016/j.actamat.2008.03.032.

Li, J.J., Weng, G.J., Chen, S.H., Wu, X.L., 2017. On strain hardening mechanism in gradient nanostructures. Int. J. Plast. 88, 89-107. https://doi.org/10.1016/j.ijplas. 2016.10.003.

Li, Y.S., Li, Y.L., Zhu, Y.T., Liu, J., 2016. A Surface Nanocrystallization Device of Metallic Materials Driven by Piezoelectricity. Chinese Patent, CN 201621380875.X.

Lin, Y., Pan, J., Zhou, H.F., Gao, H.J., Li, Y., 2018. Mechanical properties and optimal grain size distribution profile of gradient grained nickel. Acta Mater. 153, 279-289. https://doi.org/10.1016/j.actamat.2018.04.065.

Liu, X.C., Zhang, H.W., Lu, K., 2015. Formation of nano-laminated structure in nickel by means of surface mechanical grinding treatment. Acta Mater. 96, 24-36. https://doi.org/10.1016/j.actamat.2015.06.014.

Lu, K., 2014. Making strong nanomaterials ductile with gradients. Science 345, 1455-1456.

Lu, X.C., Zhang, X., Shi, M.X., Roters, F., Kang, G.Z., Raabe, D., 2019. Dislocation mechanism based size-dependent crystal plasticity modeling and simulation of gradient nano-grained copper. Int. J. Plast. 113, 52-73. https://doi.org/10.1016/j.ijplas.2018.09.007.

Lu, X.L., Lu, Q.H., Li, Y., Lu, L., 2013. Gradient confinement induced uniform tensile ductility in metallic glass. Sci. Rep. 3. https://doi.org/10.1038/srep03319.

Mahesh, S., 2006. Deformation banding and shear banding in single crystals. Acta Mater. 54, 4565-4574. https://doi.org/10.1016/j.actamat.2006.05.043.

Ming, K.S., Bi, X.F., Wang, J., 2019. Strength and ductility of CrFeCoNiMo alloy with hierarchical microstructures. Int. J. Plast. 113, 255-268. https://doi.org/10. 1016/j.ijplas.2018.10.005.

Moon, J.H., Baek, S.M., Lee, S.G., Seong, Y., Amanov, A., Lee, S., Kim, H.S., 2019. Effects of residual stress on the mechanical properties of copper processed using ultrasonic-nanocrystalline surface modification. Mater. Res. Lett. 7, 97-102. https://doi.org/10.1080/21663831.2018.1560370.

Ovid'ko, I.A., Valiev, R.Z., Zhu, Y.T., 2018. Review on superior strength and enhanced ductility of metallic nanomaterials. Prog. Mater. Sci. 94, 462-540. https://doi. org $/ 10.1016 / \mathrm{j}$.pmatsci.2018.02.002.

Pan, B., Lu, Z.X., Xie, H.M., 2010. Mean intensity gradient: an effective global parameter for quality assessment of the speckle patterns used in digital image correlation. Opt. Lasers Eng. 48, 469-477. https://doi.org/10.1016/j.optlaseng.2009.08.010.

Panin, A.V., Kazachenok, M.S., Kozelskaya, A.I., Hairullin, R.R., Sinyakova, E.A., 2015. Mechanisms of surface roughening of commercial purity titanium during ultrasonic impact treatment. Mater. Sci. Eng. A 647, 43-50. https://doi.org/10.1016/j.msea.2015.08.086.

Rupert, T.J., Gianola, D.S., Gan, Y., Hemker, K.J., 2009. Experimental observations of stress-driven grain boundary migration. Science 326, 1686-1690. https://doi. 
org $/ 10.1126 /$ science. 1178226 .

Sha, Z.D., Branicio, P.S., Lee, H.P., Tay, T.E., 2017. Strong and ductile nanolaminate composites combining metallic glasses and nanoglasses. Int. J. Plast. 90, 231-241. https://doi.org/10.1016/j.ijplas.2017.01.010.

Sun, X., Choi, K.S., Liu, W.N., Khaleel, M.A., 2009. Predicting failure modes and ductility of dual phase steels using plastic strain localization. Int. J. Plast. 25, 1888-1909. https://doi.org/10.1016/j.ijplas.2008.12.012.

Valiev, R.Z., Estrin, Y., Horita, Z., Langdon, T.G., Zehetbauer, M.J., Zhu, Y.T., 2016. Fundamentals of superior properties in bulk nanoSPD materials. Mater. Res. Lett. 4, 1-21. https://doi.org/10.1080/21663831.2015.1060543.

Wang, B., Idrissi, H., Galceran, M., Colla, M.S., Turner, S., Hui, S., Raskin, J.P., Pardoen, T., Godet, S., Schryvers, D., 2012. Advanced TEM investigation of the plasticity mechanisms in nanocrystalline freestanding palladium films with nanoscale twins. Int. J. Plast. 37, 140-156. https://doi.org/10.1016/j.ijplas.2012.04.003.

Wang, K., Tao, N.R., Liu, G., Lu, J., Lu, K., 2006. Plastic strain-induced grain refinement at the nanometer scale in copper. Acta Mater. 54, 5281-5291. https://doi.org/ 10.1016/j.actamat.2006.07.013.

Wang, X., Li, Y.S., Zhang, Q., Zhao, Y.H., Zhu, Y.T., 2017. Gradient structured copper by rotationally accelerated shot peening. J. Mater. Sci. Technol. 33, 758-761. https://doi.org/10.1016/j.jmst.2016.11.006.

Wang, Y.F., Guo, F.J., He, Q., Song, L.Y., Wang, M.S., Huang, A.H., Li, Y.S., Huang, C.X., 2019a. Synergetic deformation-induced extraordinary softening and hardening in gradient copper. Mater. Sci. Eng. A 752, 217-222. https://doi.org/10.1016/j.msea.2019.03.020.

Wang, Y.F., Huang, C.X., He, Q., Guo, F.J., Wang, M.S., Song, L.Y., Zhu, Y.T., 2019b. Heterostructure induced dispersive shear bands in heterostructured Cu. Scr. Mater. 170, 76-80. https://doi.org/10.1016/j.scriptamat.2019.05.036.

Wang, Y.F., Huang, C.X., Wang, M.S., Li, Y.S., Zhu, Y.T., 2018a. Quantifying the synergetic strengthening in gradient material. Scr. Mater. 150, 22-25. https://doi.org/ 10.1016/j.scriptamat.2018.02.039.

Wang, Y.F., Wang, M.S., Yin, K., Huang, A.H., Li, Y.S., Huang, C.X., 2019c. Yielding and fracture behaviors of coarse-grain/ultrafine-grain heterogeneous-structured copper with transitional interface. Trans. Nonferrous Metals Soc. China 29, 588-594. https://doi.org/10.1016/S1003-6326(19)64967-8.

Wang, Y.F., Yang, M.X., Ma, X.L., Wang, M.S., Yin, K., Huang, A.H., Huang, C.X., 2018b. Improved back stress and synergetic strain hardening in coarse-grain/ nanostructure laminates. Mater. Sci. Eng. A 727, 113-118. https://doi.org/10.1016/j.msea.2018.04.107.

Wei, Y.J., Li, Y.Q., Zhu, L.C., Liu, Y., Lei, X., Wang, G., Wu, Y.X., Mi, Z.L., Liu, J.B., Wang, H.T., Gao, H.J., 2014. Evading the strength-ductility trade-off dilemma in steel through gradient hierarchical nanotwins. Nat. Commun. 5, 1-8. https://doi.org/10.1038/ncomms4580.

Wu, H., Fan, G.H., Huang, M., Geng, L., Cui, X.P., Xie, H.L., 2017. Deformation behavior of brittle/ductile multilayered composites under interface constraint effect. Int. J. Plast. 89, 96-109. https://doi.org/10.1016/j.ijplas.2016.11.005.

Wu, X.L., Jiang, P., Chen, L., Yuan, F.P., Zhu, Y.T., 2014a. Extraordinary strain hardening by gradient structure. Proc. Natl. Acad. Sci. U.S.A. 111, 7197-7201. https:// doi.org/10.1073/pnas.1324069111.

Wu, X.L., Jiang, P., Chen, L., Zhang, J.F., Yuan, F.P., Zhu, Y.T., 2014b. Synergetic strengthening by gradient structure. Mater. Res. Lett. 2, 185-191. https://doi.org/10. $1080 / 21663831.2014 .935821$.

Wu, X.L., Yang, M.X., Yuan, F.P., Chen, L., Zhu, Y.T., 2016. Combining gradient structure and TRIP effect to produce austenite stainless steel with high strength and ductility. Acta Mater. 112, 337-346. https://doi.org/10.1016/j.actamat.2016.04.045.

Wu, X.L., Yang, M.X., Yuan, F.P., Wu, G.L., Wei, Y.J., Huang, X.X., Zhu, Y.T., 2015. Heterogeneous lamella structure unites ultrafine-grain strength with coarse-grain ductility. Proc. Natl. Acad. Sci. U.S.A. 112, 14501-14505. https://doi.org/10.1073/pnas.1517193112.

Wu, X.L., Zhu, Y.T., 2017. Heterogeneous materials: a new class of materials with unprecedented mechanical properties. Mater. Res. Lett. 5, 527-532. https://doi.org/ 10.1080/21663831.2017.1343208.

Yang, K., Ivanisenko, Y., Caron, A., Chuvilin, A., Kurmanaeva, L., Scherer, T., Valiev, R.Z., Fecht, H.J., 2010. Mechanical behaviour and in situ observation of shear bands in ultrafine grained Pd and Pd-Ag alloys. Acta Mater. 58, 967-978. https://doi.org/10.1016/j.actamat.2009.10.013.

Yang, M.X., Li, R.G., Jiang, P., Yuan, F.P., Wang, Y.D., Zhu, Y.T., Wu, X.L., 2019. Residual stress provides significant strengthening and ductility in gradient structured materials. Mater. Res. Lett. 7, 433-438. https://doi.org/10.1080/21663831.2019.1635537.

Yang, M.X., Pan, Y., Yuan, F.P., Zhu, Y.T., Wu, X.L., 2016. Back stress strengthening and strain hardening in gradient structure. Mater. Res. Lett. 4, 145-151. https:// doi.org/10.1080/21663831.2016.1153004.

Yuan, F.P., Yan, D.S., Sun, J.D., Zhou, L.L., Zhu, Y.T., Wu, X.L., 2019. Ductility by shear band delocalization in the nano-layer of gradient structure. Mater. Res. Lett. 7, 12-17. https://doi.org/10.1080/21663831.2018.1546238.

Zeng, Z., Li, X.Y., Xu, D.S., Lu, L., Gao, H.J., Zhu, T., 2016. Gradient plasticity in gradient nano-grained metals. Extreme Mech. Lett. 8, 213-219. https://doi.org/10. 1016/j.eml.2015.12.005.

Zhang, Z.F., Eckert, J., Schultz, L., 2003. Difference in compressive and tensile fracture mechanisms of Zr59Cu20Al10Ni8Ti3 bulk metallic glass. Acta Mater. 51, 1167-1179. https://doi.org/10.1016/S1359-6454(02)00521-9.

Zhou, H.F., Qu, S.X., Yang, W., 2013. An atomistic investigation of structural evolution in metallic glass matrix composites. Int. J. Plast. 44, 147-160. https://doi.org/ 10.1016/j.ijplas.2013.01.002.

Zhu, L.L., Lu, J., 2012. Modelling the plastic deformation of nanostructured metals with bimodal grain size distribution. Int. J. Plast. 30-31, 166-184. https://doi.org/ 10.1016/j.ijplas.2011.10.003.

Zhu, L.L., Wen, C.S., Gao, C.Y., Guo, X., Chen, Z., Lu, J., 2019. Static and dynamic mechanical behaviors of gradient-nanotwinned stainless steel with a composite structure: experiments and modeling. Int. J. Plast. 114, 272-288. https://doi.org/10.1016/j.ijplas.2018.11.005.

Zhu, Y.T., Wu, X.L., 2019. Perspective on hetero-deformation induced (HDI) hardening and back stress. Mater. Res. Lett. 7, 393-398. https://doi.org/10.1080/ 21663831.2019 .1616331

Zhu, Y.T., Wu, X.L., 2018. Ductility and plasticity of nanostructured metals: differences and issues. Mater. Today Nano 2, 15-20. https://doi.org/10.1016/j.mtnano. 2018.09.004. 